\title{
Temperature dependence of surface-melting-induced faceting of surfaces vicinal to $\mathrm{Pb}(111)$
}

\author{
H. M. van Pinxteren, B. Pluis, and J. W. M. Frenken \\ FOM-Institute for Atomic and Molecular Physics, Kruislaan 407, 1098 SJ Amsterdam, The Netherlands
}

(Received 6 December 1993)

\begin{abstract}
We present a medium-energy ion-scattering investigation of $\mathrm{Pb}$ surfaces with orientations in the [110] zone vicinal to $\mathrm{Pb}(111)$, at high temperature. Both surfaces inclined towards the (110) orientation and surfaces inclined towards the $(001)$ orientation exhibit surface-melting-induced faceting. Faceting occurs not only close to the bulk melting point, but over a range of temperatures extending at least from 589.6 $\mathrm{K}$ up to the bulk melting point $(600.7 \mathrm{~K})$. Just below the melting point, the dry facet orientation for vicinal surfaces inclined towards (110) is $3.1^{\circ} \pm 0.6^{\circ}$, the dry facet orientation towards the (001) surface is $7.3^{\circ} \pm 0.8^{\circ}$, and for both directions the melted facet orientation is approximately $14.5^{\circ}$ with respect to the (111) plane. Between 593.8 and $600.7 \mathrm{~K}$, the observed dry facet orientations vary less than $3^{\circ}$. A model calculation in terms of interfacial free energies is used to describe the facet orientations and their temperature dependence. We relate our results to recent equilibrium shape observations of $\mathrm{Pb}$ crystallites.
\end{abstract}

\section{INTRODUCTION}

Surface melting ${ }^{1-5}$ and surface faceting ${ }^{6-12}$ are wellknown phenomena. Surface melting is the formation of a liquidlike surface film at temperatures below the triple point. Faceting is the decomposition of a macroscopic surface orientation into a hill-and-valley structure of different orientations. Recently, it has been shown that the existence on a crystal of both melted and nonmelted surface orientations gives rise to the combined phenomenon: surface-melting-induced faceting. ${ }^{13-15}$ Surfaces prepared at low temperature with orientations between a melting and nonmelting orientational range decompose at high temperature into coexisting dry facets and melted facets (Fig. 1). This decomposition is an orientational phase separation similar to, e.g., the decomposition of a binary mixture.

It was Nozières who suggested that a range of surface orientations between melted and nonmelted orientations is unstable. ${ }^{16}$ With this, he explained the absence of a range of surface orientations around the (111) facet on the equilibrium shape of small $\mathrm{Pb}$ crystallites. ${ }^{17}$ Molecular-dynamics simulations by Bilalbegović, Ercolessi, and Tosatti also indicated that a coexistence of

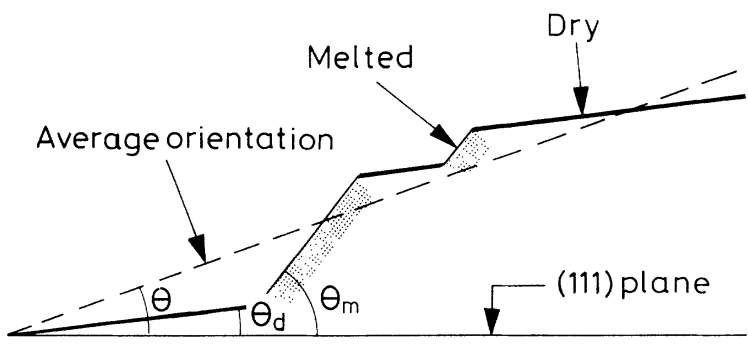

FIG. 1. Schematic picture of surface-melting-induced faceting. The surface, with initial miscut angle $\Theta$ has decomposed into dry and melted facets with orientations $\Theta_{d}$ and $\Theta_{m}$, respectively. surface-melted and nonmelted orientations causes some surface orientations to become unstable. ${ }^{13}$ In Ref. 13, a surface vicinal to $\mathrm{Pb}(111)$ was simulated, which at high temperature decomposed into two different surface orientations, a crystalline or dry surface orientation and a surface-melted orientation. The same behavior was observed for vicinal surfaces of $\mathrm{Au}(111){ }^{14}$ The authors of Refs. 13 and 14 suggested that surface-melting-induced orientational phase separation also occurs for macroscopic surfaces. The ion-scattering measurements presented in Ref. 15 have revealed that a range of surfaces misoriented with respect to the nonmelting $\mathrm{Pb}(111)$ orientation, toward the strongly melting (110) orientation, indeed exhibits surface-melting-induced faceting. At a temperature just below the bulk melting point $\left(T_{m}-0.05\right.$ $\mathrm{K})$, the measured ion intensities were shown to be a linear superposition of the signals from dry and melted parts of the surface.

In this paper we present a more extended mediumenergy ion-scattering (MEIS) study for surface orienta-

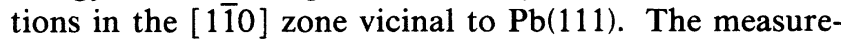
ments show that miscuts towards the (001) orientation also result in surface-melting-induced faceting, albeit with facet orientations different from those for miscuts toward (110). In addition, we demonstrate that the faceting phenomenon is not restricted to temperatures close to $T_{m}$; at $10 \mathrm{~K}$ below $T_{m}$ the measurements are still indicative of faceting, the facet orientations being almost the same as at higher temperatures. In Sec. II we introduce the thermodynamics of surface melting in terms of interfacial free energies, and we explain how the orientation dependence of these energies gives rise to surfacemelting-induced faceting. Section III describes the application of MEIS to a surface which has decomposed into coexisting dry and melted facets. The procedure of analysis of the measured energy spectra is described in Sec. IV, and the results of the analysis, i.e., the measured orientations of the stable dry and melted facets, are presented in Sec. V. Using low-temperature measurements of the surface free energy of $\mathrm{Pb}$, we predict the 
facet orientations and their temperature dependence in Sec. VI. The results of our calculation agree with the experimental results. In Sec. VII we discuss the hightemperature equilibrium shapes reported for $\mathrm{Pb}$ (Refs. 17 and 18) in terms of surface-melting-induced faceting. Other work related to the surface-melting-induced faceting of surfaces vicinal to $\mathrm{Pb}(111)$ is discussed in Sec. VIII.

\section{SURFACE-MELTING-INDUCED FACETING}

Surface melting has been observed on many surfaces of, e.g., metals and noble-gas crystals. ${ }^{1-4}$ On these surfaces a liquidlike film forms which diverges in thickness as the temperature approaches the bulk melting temperature $T_{m}$. If we do not allow any faceting, we can predict whether or not a given crystal face melts on the basis of the difference in the Gibbs free energy at $T_{m}$ between the dry surface and the surface covered by a macroscopically thick liquidlike film,

$$
\Delta \gamma=\gamma_{\mathrm{sv}}-\gamma_{\mathrm{sl}}-\gamma_{\mathrm{lv}}
$$

where $\gamma_{\mathrm{sv}}, \gamma_{\mathrm{sl}}$, and $\gamma_{\mathrm{lv}}$ are the free energies of the solidvapor, solid-liquid, and liquid-vapor interfaces, respectively. The occurrence of surface melting depends on surface orientation through the orientation dependence of the free-energy difference $\Delta \gamma(\Theta)$. For the melting surfaces $\Delta \gamma(\Theta)$ is positive, whereas it is negative for closepacked surfaces such as $\mathrm{Pb}(111)$ and $\mathrm{Al}(111)$, which do not melt. ${ }^{1,2,4,19}$ Recent experiments have shown that the sign of $\Delta \gamma(\Theta)$ does not account for all surface-melting phenomena. First, measurements on $\mathrm{Pb}(001)$ (Ref. 20) and $\mathrm{Ge}(111)$ (Ref. 21) have demonstrated that these surfaces exhibit incomplete melting. They develop a disordered film with a thickness which remains finite up to the highest temperatures. The incomplete melting of these surfaces results from layering ${ }^{22}$ or metallization ${ }^{23}$ effects. Second, for certain vicinal orientations between melting and nonmelting orientational ranges, it is not sufficient to consider the surface free energies for each orientation separately. ${ }^{15}$ One has to take into account the surface free energies for all surrounding orientations as well. In an earlier analysis of the melting of $\mathrm{Pb}$ surfaces, ${ }^{2}$ it was implicitly assumed that a macroscopic surface retains its orientation down to the atomic scale. In the following paragraph we show that this assumption is not justified. For some surface orientations it is energetically favorable to break up into a collection of dry and melted facets with other orientations (Fig. 1), despite the fact that this faceting enlarges the total surface area.

In general, faceting occurs when the so-called $\beta$ plot of the surface free energy exhibits a concave region. ${ }^{6,8}$ In a $\beta$ plot, the surface free energy is expressed per unit area projected on a reference plane, in order to account for the energy cost of creating extra area by changing the local surface slope. The projected energy is plotted as a function of the surface slope. When the $\beta$ plot contains a concave region, a range of orientations is unstable and surfaces with an orientation in this range phase separate into two (or three) stable facets. The orientations of the stable facets are determined by a double-tangent construction, ${ }^{7,8}$ which is analogous to the classic Maxwell con- struction. Usually, faceting is a low-temperature phenomenon which is caused by, e.g, the presence of adsorbates $^{9,10}$ which favor particular crystal faces, or the existence of surface reconstructions which lower the free energy of particular orientations. ${ }^{11,12,24-26}$ By contrast, surface-melting-induced faceting occurs at high temperatures.

Nozières was the first to realize that the phenomenon of surface melting causes certain surface orientations to become unstable. ${ }^{16}$ Figure 2 is a $\beta$ plot of the orientation dependence of the free energy for dry surfaces (dashdotted curve) and that for melted surfaces (dashed curve) in the vicinity of the nonmelting $\mathrm{Pb}(111)$ orientation at the bulk melting temperature $T_{m}$ (see also Sec. VI). The lowest-energy choice seems to be the dry state for orientations close to (111) and the melted state for orientations beyond the intersection of the two energy curves. However, the crossing leads to effectively concave sections in the lowest-energy curve. The concavity was used by Nozières to explain qualitatively the absence of a range of surface orientations on the equilibrium shape of small $\mathrm{Pb}$ crystallites which had been measured by Heyraud and Métois. ${ }^{17}$ Nozières did not discuss macroscopic surfaces. The instability of certain orientations on the equilibrium shape implies that a macroscopic surface prepared with such an orientation should lower its energy by faceting: ${ }^{6}$ surface-melting-induced faceting.

The double-tangent lines in Fig. 2 identify two ranges of unstable surface orientations: between $\Theta_{d^{+}}$and $\Theta_{m}{ }^{+}$,

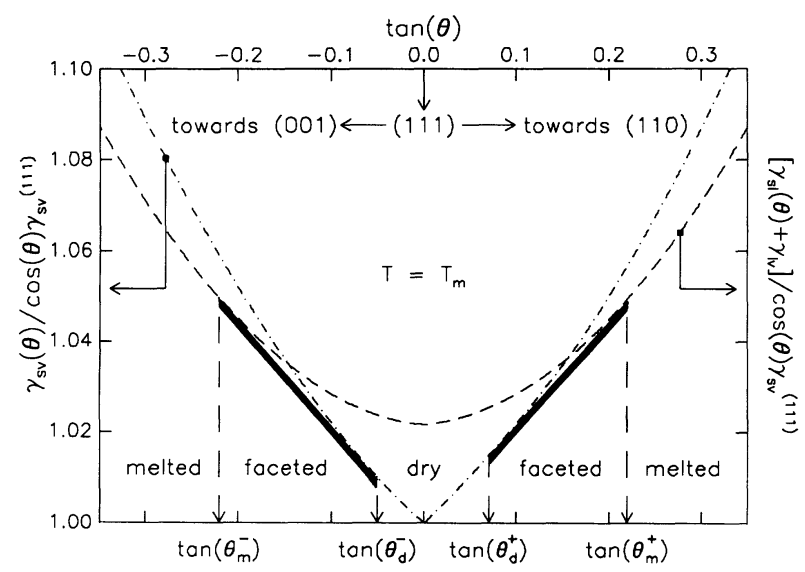

FIG. 2. $\beta$ plot of the normalized surface free energies (Refs. 2 and 31) as a function of surface slope, $\tan (\Theta)$, around $\mathrm{Pb}(111)$ at $T_{m}$. The dash-dotted curve shows the free energy $\gamma_{\mathrm{sv}}(\Theta) / \cos (\Theta) \gamma_{\mathrm{sv}}^{(111)}$ for dry surfaces, and the dashed curve the sum $\left\{\left[\gamma_{\mathrm{sl}}(\Theta)+\gamma_{\mathrm{lv}}\right] / \cos (\Theta)\right\} \gamma_{\mathrm{sv}}^{(111)}$ for melted surfaces at the melting point. The energies have been expressed per unit area projected on the (111) plane by the division by $\cos (\Theta)$. Both curves have been normalized to the energy $\gamma_{\mathrm{sv}}^{(111)}$ for the dry (111) orientation. The double-tangent construction, indicated by the two solid-line segments, indicates that all orientations with miscut angles between $\Theta_{d}+$ and $\Theta_{m}+$ (or between $\Theta_{d^{-}}$and $\left.\Theta_{m}{ }^{-}\right)$lower their free energy by decomposing into the dry orientation $\Theta_{d^{+}}$(or $\Theta_{d^{-}}$) and the surface-melted orientation $\Theta_{m}+\left(\right.$ or $\Theta_{m}-$ ). 
and between $\Theta_{d^{-}}$and $\Theta_{m}{ }^{-}$. A macroscopic surface with an orientation $\Theta$ in one of these two ranges should exhibit surface-melting-induced faceting into a hill-and-valley structure of dry facets with orientation $\Theta_{d}$ and melted facets with orientation $\Theta_{m}$. The fraction of projected surface area which is melted, $F_{m}$, and the fraction which is dry, $1-F_{m}$, are given by the lever rule ${ }^{27}$

$$
\tan (\Theta)=F_{m} \tan \left(\Theta_{m}\right)+\left(1-F_{m}\right) \tan \left(\Theta_{d}\right) .
$$

This lever rule expresses the condition that the average slope of the macroscopic surface remains $\tan (\Theta)$.

The hill-and-valley structure is fully equilibrated only when the entire surface has rearranged into two large facets: one dry and one melted facet. In practice, the size of the facets is limited by diffusion kinetics. Furthermore, impurities and crystal defects may pin the facet edges.

\section{MEIS APPLIED TO SURFACE-MELTING-INDUCED FACETING}

The sample was a cylindrically shaped $\mathrm{Pb}$ crystal (dimensions $12 \times 12 \times 5 \mathrm{~mm}^{3}$ ) exposing a range of orientations in the [110] zone vicinal to the (111) face (Fig. 3). We define the vicinal orientation angles $\Theta$ with respect to the (111) plane, such that $\Theta$ is negative for vicinal surfaces with a miscut toward the (001) face, and positive for surfaces with a miscut toward (110). ${ }^{28}$ For details concerning the sample preparation and the temperature control, we refer to Ref. 2 .

The technique of medium-energy ion scattering (MEIS) and its application to surface melting have been described in detail in Refs. 3 and 29. Here we discuss how surfacemelting-induced faceting was observed with MEIS. A $75.6-\mathrm{keV}$ proton beam was aligned with the [ $\overline{10} \overline{1}]$ axis of the crystal, and backscattered protons were detected along the [1 21$]$ direction (Fig. 3). By translating the

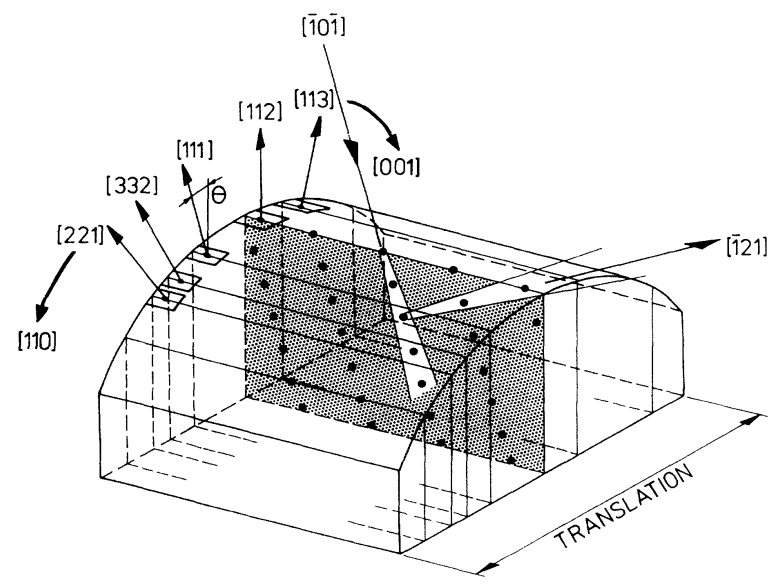

FIG. 3. The shadowing and blocking geometry in the (111) scattering plane of a cylindrical single crystal of $\mathrm{Pb}$. The crystal exposes a range of surface orientations around the (111) orientation. Surfaces with a positive orientation angle $\Theta$ are miscut, with respect to (111), toward the (110) orientation, whereas surfaces with a negative $\Theta$ are oriented toward $(001)$. crystal normal to the scattering plane, we could explore a range of surface orientations under identical conditions of preparation, temperature, and scattering geometry. The backscattered protons were counted as a function of their energy with a resolution of $300 \mathrm{eV}$. In our scattering geometry, this energy resolution corresponds to a depth resolution of $7 \AA$ [the electronic stopping power for 75.6-keV protons in $\mathrm{Pb}$ is $42.7 \mathrm{eV}$ per $10^{15}$ atoms $\mathrm{cm}^{-2}$ (Ref. 30)].

The shadowing and blocking effects in this aligned geometry allow a direct distinction between a crystalline (dry) surface and a disordered (melted) surface film. For a dry surface, the energy spectrum shows a narrow peak at high energy, containing the scattering signal of the outermost monolayers. Atoms in deeper layers are hidden in the shadow of the ordered surface layers and therefore contribute only a low backscattered intensity at energies below the surface peak. This is illustrated by Fig. 4, which displays the energy spectra obtained at room temperature. At room temperature, all $\mathrm{Pb}$ surface orientations are crystalline. Hence all the energy spectra are alike. There is a slight increase in the area of the surface peak in the energy spectrum, i.e., of the number of atoms per unit area visible to proton beam and detector, with increasing miscut angle. The same effect has been observed for surfaces vicinal to $\mathrm{Pb}(001),{ }^{20}$ and it is probably due to a relaxation of step atoms. By contrast, at high temperatures, the measured energy spectra show a strong variation with surface orientation. Figure 5(a) displays energy spectra of protons backscattered from a range of vicinal surfaces at a temperature of $600.65 \mathrm{~K}$. The $\mathrm{Pb}(111)$ surface is crystalline up to $T_{m}(600.7 \mathrm{~K})$; its energy spectrum [Fig. 5(a), open diamonds] shows a narrow peak at high energy containing the scattering signal of the outermost three monolayers. A miscut angle of $19.2^{\circ}$ with respect to the (111) face results in quite a different spectrum (open triangles). At high energies the backscattered yield is constant at the value expected for disordered $\mathrm{Pb}$, over an energy range corresponding to a

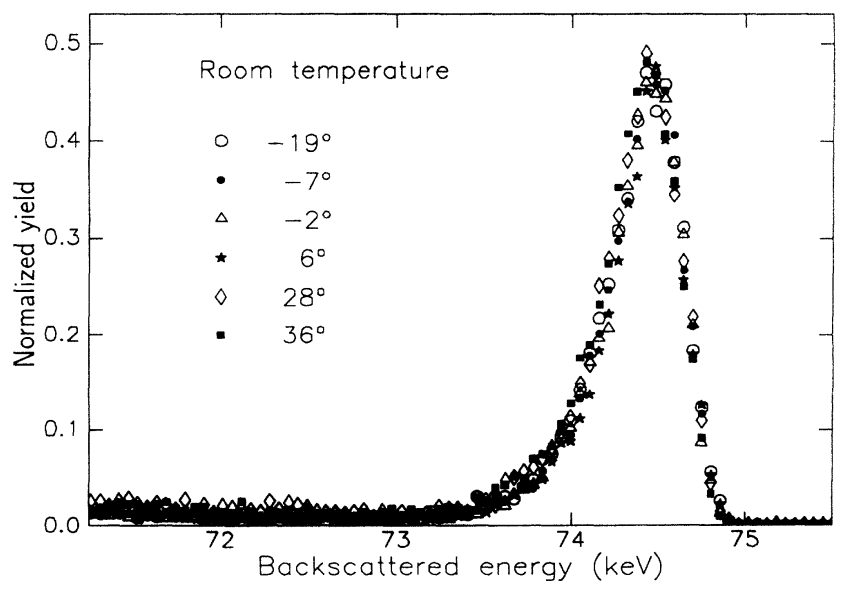

FIG. 4. Energy spectra obtained at room temperature in the scattering geometry of Fig. 3, for different surface orientations $\Theta$. The backscattering yield has been normalized to the random height. 
melted film with a thickness of approximately $1.7 \mathrm{~nm}$. In the crystalline interior below this melted surface film, i.e., at lower backscattered energies, the shadowing and blocking effects rapidly reduce the proton yield.

The shapes of the energy spectra obtained for miscut angles of $6.0^{\circ}, 9.1^{\circ}$, and $12.3^{\circ}$ are again completely different [Fig. 5(a)]. They resemble neither the sharp peak expected for a dry surface nor the shape expected

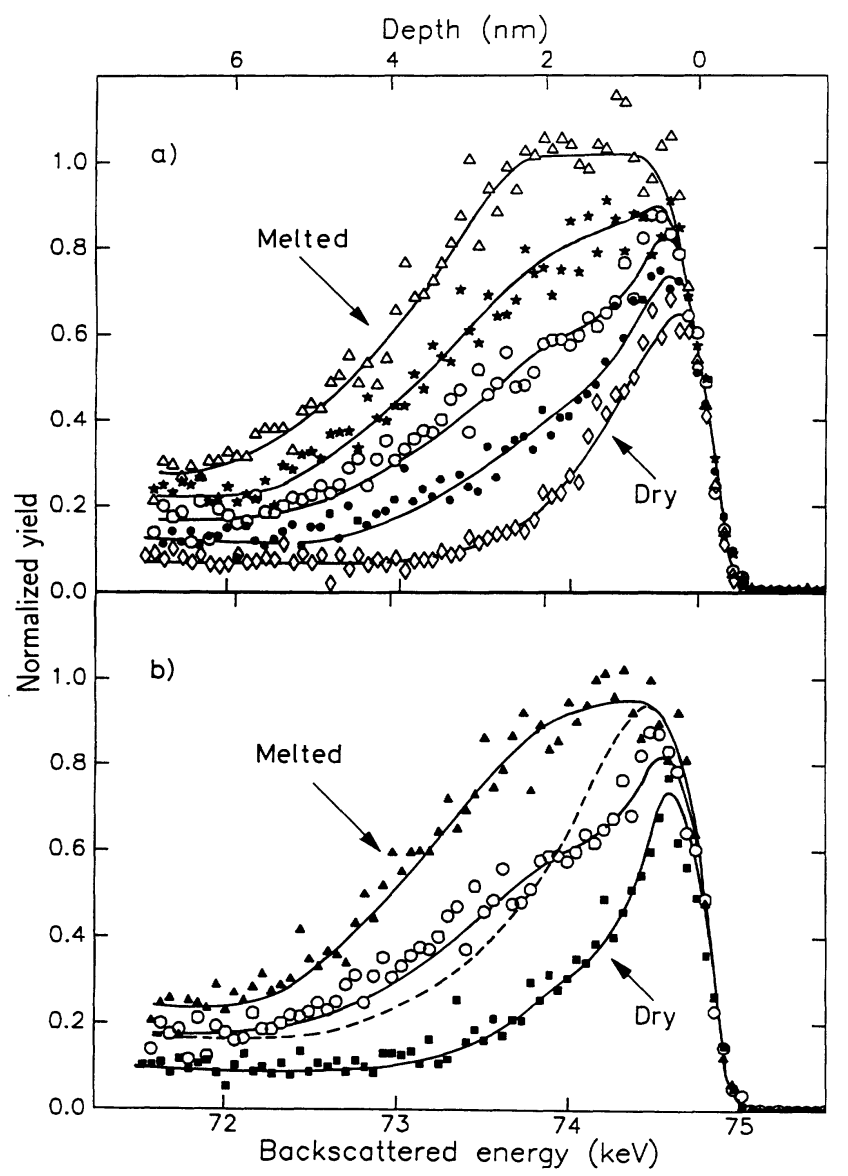

FIG. 5. (a) Energy spectra of protons backscattered from $\mathrm{Pb}(111)$, and vicinal surfaces with miscut angles toward (110) measured at $600.65 \mathrm{~K}$. On their way through the $\mathrm{Pb}$, the protons lose energy due to the electronic stopping (Ref. 30). The energy loss is proportional to the path length and thus to the depth from which the protons are backscattered. The depth scale is indicated at the top of the figure. The backscattered intensities have been normalized to yield a value of 1.0 for a melted film. The figure shows spectra obtained on $\mathrm{Pb}(111)(\diamond)$ and on surfaces with miscut angles of $6.0^{\circ}(\bullet), 9.1^{\circ}(O), 12.3^{\circ}(\star)$, and $19.2^{\circ}(\triangle)$. The solid curves serve to guide the eye. The peak shapes indicate that the (111) surface is dry and that the $19.2^{\circ}$-vicinal surface is covered with a melted film of approximately $1.7 \mathrm{~nm}$. The other three orientations are faceted. (b) Energy spectra for the (predominantly) dry $2.9^{\circ}(\square)$, melted $15.7^{\circ}$ $(\Delta)$, and faceted $9.1^{\circ}(O)$ orientations at $600.65 \mathrm{~K}$. The dashed curve is the expected spectrum shape if the $9.1^{\circ}$ vicinal surface were covered with a continuous liquidlike film. The middle solid curve is a linear combination of $50 \%$ of the $2.9^{\circ}$ and $50 \%$ of the $15.7^{\circ}$ spectrum, illustrating that the $9.1^{\circ}$ surface consists of coexisting dry and melted facets. for a surface covered with a melted film of uniform thickness. The energy spectrum expected for a vicinal orientation which has decomposed into coexisting dry and melted facets (Fig. 1) is a linear combination of the spectrum for a melted surface and the spectrum for a dry surface. Figure 5(b) illustrates that the spectrum obtained for the 9. $1^{\circ}$ vicinal surface at $600.65 \mathrm{~K}$ is indeed a linear combination of a narrow spectrum, typical of a dry surface, and a broad spectrum typical of a melted surface. The dashed curve in Fig. 5(b) indicates what the energy spectrum of the $9.1^{\circ}$ vicinal orientation would look like if that surface were covered with a continuous liquidlike film. Indeed, the linear combination (center solid curve) of $50 \%$ of the $2.9^{\circ}$ spectrum and $50 \%$ of the $15.7^{\circ}$ spectrum fits the $9.1^{\circ}$ data much better than the dashed curve.

\section{DATA ANALYSIS}

In this section we describe how the facet orientations $\Theta_{d^{+}}, \Theta_{d^{-}}, \Theta_{m}{ }^{+}$, and $\Theta_{m^{-}}$are obtained from the measured energy spectra. We start by demonstrating the adopted procedure for spectra obtained for surfaces with a positive miscut angle at $600.65 \mathrm{~K}$. We have measured energy spectra for vicinal surfaces with orientation angles of $0.0^{\circ}, 2.9^{\circ}, 6.0^{\circ}, 9.1^{\circ}, 12.3^{\circ}, 15.7^{\circ}$, and $19.2^{\circ}$ (Fig. 5). We fit each energy spectrum, measured for orientation $\Theta$, by all linear combinations of two spectra measured for orientations $\Theta_{\text {low }}$ and $\Theta_{\text {high }}$, with $\left|\Theta_{\text {low }}\right| \leq|\Theta| \leq\left|\Theta_{\text {high }}\right|$. For each combination of $\Theta_{\text {low }}$ and $\Theta_{\text {high }}$, the best fit to the spectrum for orientation $\Theta$ is obtained for a fraction $F_{\text {high }}$ of the $\Theta_{\text {high }}$ spectrum and a fraction $\left(1-F_{\text {high }}\right)$ of the $\Theta_{\text {low }}$ spectrum. In Fig. 6 the obtained fractions $F_{\text {high }}$ (circles) have been plotted as a function of $\tan \left(\Theta+19.5^{\circ}\right)$, for all possible combinations of $\Theta_{\text {low }}$ and $\Theta_{\text {high }}$. The statistical error in the fit results is smaller than the size of the plotting symbols. The statistical accuracy is determined by the number of counts in the individual energy spectra and by the magnitude of the difference between the low$\left(\Theta_{\text {low }}\right)$ and high-angle $\left(\Theta_{\text {high }}\right)$ spectra. For a surface with orientation $\Theta$ which has decomposed into melted facets with orientation $\Theta_{m}$ and dry facets with orientation $\Theta_{d}$, the fractions of melted and dry areas are dictated by the lever rule [Eq. (2)]. As a result, the fraction $F_{m}$ of the melted energy spectrum in the spectrum for a faceted vicinal is also dictated by this lever rule. In our scattering geometry, the number of visible atoms is measured per unit area projected onto the (112) plane, which makes an angle of $19.5^{\circ}$ with the (111) plane (Fig. 7). Therefore, we use the (112) orientation as a reference plane. Hence the lever rule, i.e., the condition that the average surface orientation remains equal to the initial miscut angle $\Theta$, is given by

$$
\begin{aligned}
\tan \left(\Theta+19.5^{\circ}\right)= & F_{m} \tan \left(\Theta_{m}+19.5^{\circ}\right) \\
& +\left(1-F_{m}\right) \tan \left(\Theta_{d}+19.5^{\circ}\right) .
\end{aligned}
$$

$F_{m}$ increases linearly in $\tan \left(\Theta+19.5^{\circ}\right)$, from 0 to 1 , for $\Theta$ running from $\Theta_{d}$ to $\Theta_{m}$. It is easily verified that for all combinations of $\Theta_{\text {low }}$ and $\Theta_{\text {high }}$ with $\left|\Theta_{d}\right| \leq\left|\Theta_{\text {low }}\right|$ $<\left|\Theta_{\text {high }}\right| \leq\left|\Theta_{m}\right|$, the fraction $F_{\text {high }}$ also increases linearly in $\tan \left(\Theta+19.5^{\circ}\right)$ from 0 to 1 , when $\Theta$ runs from $\Theta_{\text {low }}$ to 


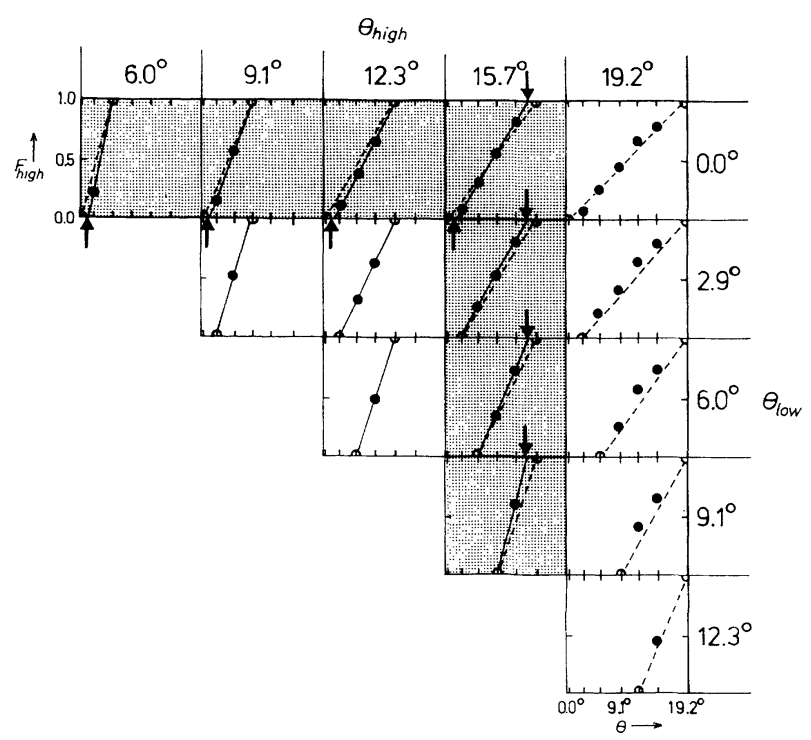

FIG. 6. Results of fitting the energy spectra measured at $600.65 \mathrm{~K}$ for positive miscut angles by a linear combination of two other spectra. For each combination of $\Theta_{\text {low }}$ and $\Theta_{\text {high }}$ (one panel in the table) the best fits to the spectra for orientations $\Theta$ between those angles are obtained for the fractions $F_{\text {high }}$ (solid circles) of the high-angle spectrum. The open circles indicate the trivial result of $F_{\text {high }}=0$ for $\Theta=\Theta_{\text {low }}$, and $F_{\text {high }}=1$ for $\Theta=\Theta_{\text {high }}$. The fractions for each combination of $\Theta_{\text {low }}$ and $\Theta_{\text {high }}$ have been plotted as a function of $\tan \left(\Theta+19.5^{\circ}\right.$ ) (see text and Fig. 7), on a scale ranging from $\tan \left(0^{\circ}+19.5^{\circ}\right)$ to $\tan \left(19.2^{\circ}+19.5^{\circ}\right)$. Spectra have been measured for $0.0^{\circ}, 2.9^{\circ}$, $6.0^{\circ}, 9.1^{\circ}, 12.3^{\circ}, 15.7^{\circ}$, and $19.2^{\circ}$, corresponding to the tick marks on the horizontal axes. The geometrical condition [Eq. (3)] is indicated by the dashed straight lines. The solid straight lines are fits to the fractions obtained for orientations within the range of surface-melting-induced faceting. The arrows pointing up locate the orientation of the stable dry facets $\Theta_{d}$, and the arrows pointing down locate the orientation of the melted facets $\Theta_{m}$. The shading of the panels with $\Theta_{\text {low }}=0.0^{\circ}$ and $\Theta_{\text {high }}=15.7^{\circ}$ is discussed in the text.

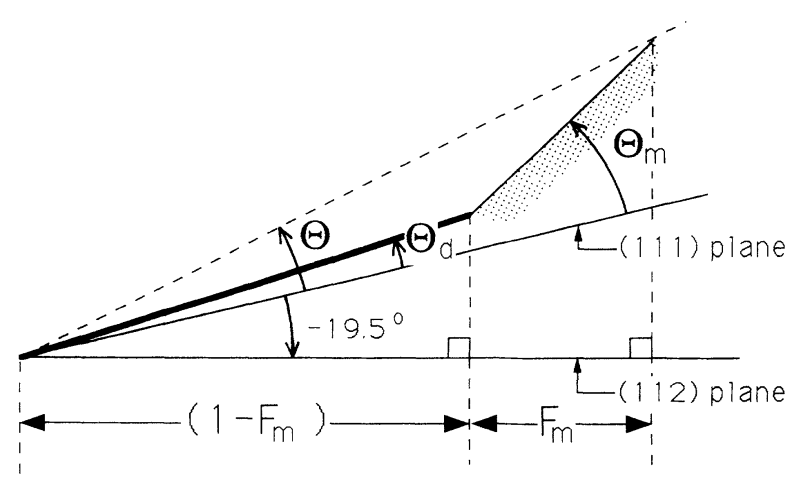

FIG. 7. Illustration of the lever rule [Eq. (3)], which expresses that the average orientation of a faceted surface is fixed. Indicated are the fraction of melted surface area projected on the (112) plane, $F_{m}$, and the fraction of dry surface area projected on the (112) plane, $1-F_{m}$. In the fitting of the MEIS spectra this is the relevant projection, since the scattering plane was perpendicular to the (112) plane.
$\Theta_{\text {high }}$. We find $\Theta_{d}+$ simply by decreasing $\Theta_{\text {low }}$ until the required linearity no longer holds. Similarly, $\Theta_{m}+$ is obtained by increasing $\Theta_{\text {high }}$ until the linearity is again violated. This procedure is illustrated in Fig. 6. In each panel, the dashed line is the linear behavior expected from the lever rule [Eq. (3)]. For $2.9^{\circ} \leq \Theta_{\text {low }}$ $<\Theta_{\text {high }} \leq 12.3^{\circ}$ (three panels in the center) the fractions $F_{\text {high }}$ agree very well with this expected linearity. All surfaces with orientations in this range can be described as a linear combination of two other surfaces in this range. For $\Theta_{\text {low }}=0.0^{\circ}$ and $\Theta_{\text {high }} \leq 12.3^{\circ}$ (horizontal row of shaded panels), all fractions $F_{\text {high }}$ are too low with respect to the dashed line. This implies that $\Theta_{d^{+}}$is between $0.0^{\circ}$ and $2.9^{\circ}$. In fact, the fractions in the panels in the top row follow alternative (solid) straight lines, which all extrapolate to $F_{\text {high }}=0$ at $\Theta \approx 2^{\circ}$ (arrows pointing up). We conclude that $\Theta_{d^{+}}=2.0^{\circ} \pm 0.9^{\circ}$. In the same way we determine $\Theta_{m}+$. For $\Theta_{\text {high }}=15.7^{\circ}$ and $\Theta_{\text {low }} \geq 2.9^{\circ}$ (vertical shaded column), all fractions $F_{\text {high }}$ are too high with respect to the dashed lines. From this we know that $\Theta_{m}{ }^{+}$ lies between $12.3^{\circ}$ and $15.7^{\circ}$. For $\Theta_{\text {high }}=15.7^{\circ}$, the fractions follow the (solid) straight lines, which all extrapolate to $F_{\text {high }}=1$ at $\Theta \approx 14.5^{\circ}$ (arrows pointing down). We conclude that $\Theta_{m}{ }^{+}=14.5^{\circ} \pm 0.9^{\circ}$. The error margins in the orientations $\Theta_{d^{+}}$and $\Theta_{m}+$ result from the statistical accuracy of the fits and from the precision of our calibration of the probed surface orientation as a function of the beam position on the crystal. We estimate the latter to be $\pm 0.8^{\circ}$.

The method for finding $\Theta_{d}$ and $\Theta_{m}$ in the region with miscut angles toward (001) or at another temperature is the same as the one described above. As a second example, Fig. 8 displays the results from fitting the energy spectra which were obtained at $593.8 \mathrm{~K}$ for surfaces with orientations $-3.0^{\circ},-5.8^{\circ},-8.7^{\circ},-11.5^{\circ}$, and $-14.3^{\circ}$. The statistical error in these fit results, indicated by the vertical bars, is larger than for the results at $600.65 \mathrm{~K}$ because the differences between the spectra for different

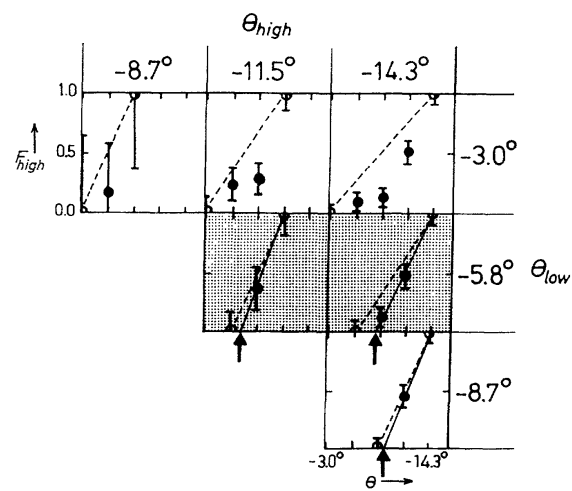

FIG. 8. Results from fitting the energy spectra measured at $593.8 \mathrm{~K}$ for negative miscut angles. The layout of this figure corresponds to that of Fig. 6. Spectra have been measured for $-3.0^{\circ},-5.8^{\circ},-8.7^{\circ},-11.5^{\circ}$, and $-14.3^{\circ}$. The geometrical condition is again indicated by the dashed straight line. The error bars depict the statistical accuracy in the fractions $F_{\text {high }}$ obtained from fitting the energy spectra with each other. 
orientations are smaller at lower temperature. The fraction obtained by fitting the $-11.5^{\circ}$ spectrum with a linear combination of the spectra measured for $\Theta_{\text {low }}=-8.7^{\circ}$ and $\Theta_{\text {high }}=-14.3^{\circ}$ (lower right panel), agrees with the linearity imposed by the condition that the average surface orientation has to be maintained [dashed line; see Eq. (3)]. For $\Theta_{\text {low }}=-3.0^{\circ}$ (horizontal top row of panels) the linearity which is predicted for all combinations of $\Theta_{\text {low }}$ and $\Theta_{\text {high }}$, if $\left|\Theta_{d}\right| \leq\left|\Theta_{\text {low }}\right|<\left|\Theta_{\text {high }}\right| \leq\left|\Theta_{m}\right|$ is clearly violated. This implies that for vicinal surfaces with negative miscut angles at $T=593.8 \mathrm{~K}$, the orientation of the dry facet, $\Theta_{d^{-}}$, has a miscut angle of more than $3.0^{\circ}$ with respect to the (111) plane. The fit results obtained with $\Theta_{\text {low }}=-5.8^{\circ}$ (shaded panels) lie below the straight line predicted by Eq. (3), suggesting that even $-5.8^{\circ}$ is outside the orientation region where surface-melting-induced faceting occurs. By again extrapolating the alternative (solid) straight lines which follow from the fractions $F_{m}$, we find $\Theta_{d^{-}}=-8.2^{\circ} \pm 1.4^{\circ}$. Figure 8 shows that the range of vicinal orientations with negative miscut angles which has been measured at $593.8 \mathrm{~K}$ is not wide enough to determine $\Theta_{m}-$. For the magnitude of $\Theta_{m}$, we can only give a lower limit: $\Theta_{m}-\leq-14.3^{\circ} \pm 0.8^{\circ}$.

\section{TEMPERATURE DEPENDENCE OF THE FACETING}

The results of the analysis of the entire data set are plotted as a function of temperature in Fig. 9. The solid and dashed curves in this figure are discussed in Sec. VI.

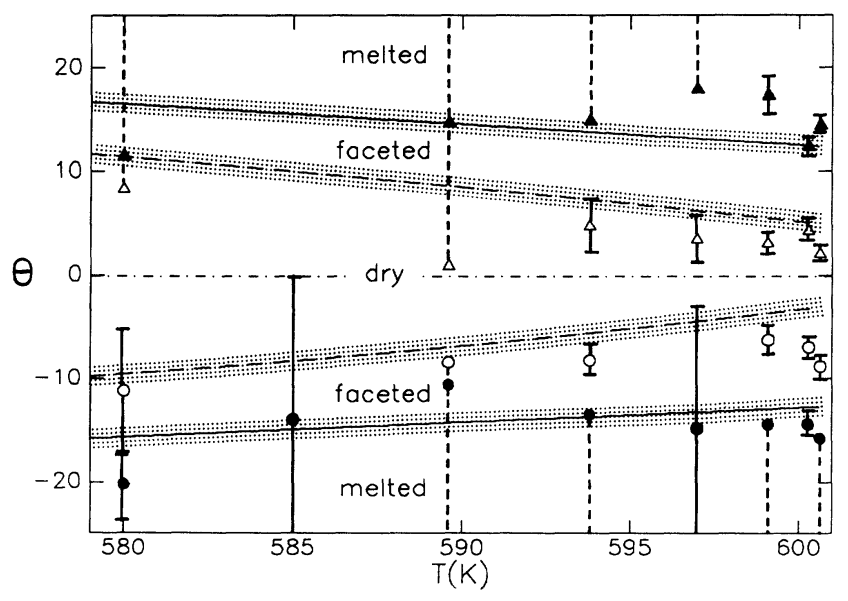

FIG. 9. Experimentally determined facet orientation angles as a function of temperature. The triangles are the results for vicinals with a miscut toward the (110) orientation, the circles for vicinals with a miscut toward the (001) orientation. Open symbols depict the dry facet orientations, solid symbols the melted facet orientations. Dashed error bars indicate that the depicted angle is either a lower or an upper limit for the facet orientation. The curves are part of the model-calculation results of Sec. VI (see Fig. 11). The dashed curves correspond to the predictions for the dry facet angles, the solid curves to those for the melted facet angles. The shading around these curves indicates the estimated accuracy of the model.
The open symbols depict the values obtained for $\Theta_{d}$, the solid symbols are the results for $\Theta_{m}$. For some cases, only upper or lower limits for the facet orientations could be determined. In those cases the plotting symbols represent the limiting values and they are marked by dashed error bars. At $589.6 \mathrm{~K}$, we investigated a range of orientations, $-16^{\circ}$ to $+15^{\circ}$, that was not wide enough to yield other than lower limits for $\Theta_{d^{+}}$and $\Theta_{m}+$, and upper limits for $\Theta_{d^{-}}$and $\Theta_{m^{-}}$. At still lower temperatures, the number of investigated orientations between $-30^{\circ}$ and $+30^{\circ}$ was very low, so that we cannot determine the facet orientations from the fitting results. For 580 and $585 \mathrm{~K}$, we can only conclude from the data that if surface-melting-induced faceting occurs, it takes place in the indicated angular ranges.

Figure 9 demonstrates that surface melting induces faceting, not only close to the melting point but over an appreciable temperature range, which runs at least from 589.6 to $600.65 \mathrm{~K}$. At all these temperatures, a range of nonmelting orientations around (111) remains stable against faceting. The temperature dependence of the dry facet orientations is rather weak. Despite the large error bars, the data show that between $593.8 \mathrm{~K}$ and $T_{m}$ the dry facet orientations are constant within $3^{\circ}$ for both miscut directions. Since the temperature dependence of the facet orientations is so weak, we average the facet angles for the three highest experimental temperatures, 599.1, 600.3 , and $600.65 \mathrm{~K}$, in order to obtain accurate values for the facet angles close to the melting point. We obtain $\Theta_{d^{+}}\left(T_{m}\right)=3.1^{\circ} \pm 0.6^{\circ}, \Theta_{d^{-}}\left(T_{m}\right)=-7.3^{\circ} \pm 0.8^{\circ}, \Theta_{m^{+}}\left(T_{m}\right)$ $=14.7^{\circ} \pm 1.4^{\circ}$, and $\Theta_{m}-\left(T_{m}\right)=-14.5^{\circ} \pm 1.2^{\circ}$.

\section{MODEL CALCULATION OF THE FACETING}

In this section we calculate the temperature dependence of the facet orientations $\Theta_{d^{+}}, \Theta_{d^{-}}, \Theta_{m}+$, and $\Theta_{m^{-}}$. To this end, we make use of the orientation dependence of the free energy for dry $\mathrm{Pb}$ surfaces, which has been determined from the equilibrium shape of small $\mathrm{Pb}$ particles. ${ }^{31}$ We calculate the temperature dependence of the effective free energy for melted surfaces using a Landau model, ${ }^{5}$ and apply the double-tangent construction to the free energies for dry and melted surfaces to determine the stable facet orientations $\Theta_{d}$ and $\Theta_{m}$. Finally, the calculation results are compared to our experimental results for the facet orientations as a function of temperature.

Figure 2 shows the $\beta$ plot of the relative interfacial free energies for $\mathrm{Pb}$ at the bulk melting temperature, normalized to the free energy for the dry (111) surface, $\gamma_{\mathrm{sv}}^{(111)}$. The dash-dotted curve $\gamma_{\mathrm{sv}}(\Theta) / \cos (\Theta) \gamma_{\mathrm{sv}}^{(111)}$, is the normalized free energy for the solid-vapor interface, and the dashed curve $\left[\gamma_{\mathrm{sl}}(\Theta)+\gamma_{\mathrm{lv}}\right] / \cos (\Theta) \gamma_{\mathrm{sv}}^{(111)}$, is the normalized energy for melted surfaces, which, for large film thicknesses, is equal to the sum of the free energy for the solid-liquid interface and the free energy for the liquidvapor interface. $\gamma_{\mathrm{sv}}(\Theta) / \gamma_{\mathrm{sv}}^{(11)}$ has been determined from equilibrium shape measurements by Heyraud and Métois $^{31}$ at 473 K. At higher temperatures, $\gamma_{\text {sv }}(\Theta) / \gamma_{\text {sv }}^{(111)}$ is not accessible via equilibrium shape mea- 
surements because some $\mathrm{Pb}$ surface orientations start to melt around $500 \mathrm{~K} \cdot{ }^{1-3}$ We assume that the orientation dependence of $\gamma_{\mathrm{sv}}(\Theta) / \gamma_{\mathrm{sv}}^{(111)}$ remains unchanged between $473 \mathrm{~K}$ and $T_{m}$. For $\left[\gamma_{\mathrm{sl}}(\Theta)+\gamma_{\mathrm{lv}}\right] / \gamma_{\mathrm{sv}}^{(111)}$, we use the results of Ref. 2 , where the best fit to measured melted-film thicknesses was obtained for $\gamma_{1 \mathrm{v}}=0.501 \mathrm{~J} / \mathrm{m}^{2}$, with the assumption $\gamma_{\mathrm{sl}}(\Theta)=0.1 \gamma_{\mathrm{sv}}(\Theta)$, and for a fixed choice for $\gamma_{\mathrm{sv}}^{(111)}\left(T_{m}\right)$ of $0.544 \mathrm{~J} / \mathrm{m}^{2}$. ${ }^{32}$

In order to predict the temperature dependence of the facet orientations $\Theta_{d}$ and $\Theta_{m}$, we have to know the temperature dependence of the differences between the surface free energies. We start with the expression, obtained from Landau theory, ${ }^{5}$ for the effective surface free energy of a melted surface. The effective free energy $\gamma_{\mathrm{slv}}(\Theta, T)$ per unit area of a surface with orientation $\Theta$, wetted by a liquidlike film with $N$ positionally disordered atoms per unit area at temperature $T$, is, in the limit of large $N$, given by

$$
\begin{aligned}
\gamma_{\mathrm{slv}}(N, \Theta, T)= & \gamma_{\mathrm{sl}}(\Theta)+\gamma_{\mathrm{lv}}+N L_{m}\left(1-T / T_{m}\right) \\
& +\Delta \gamma(\Theta) e^{-N / N_{0}},
\end{aligned}
$$

where $L_{m}=7.93 \times 10^{-21} \mathrm{~J}$ is the latent heat of melting per atom, and $N_{0}=\rho_{1} \xi / 2$ is a characteristic decay constant which depends on the correlation length within the liquid phase, $\xi$, and the atomic density in the liquid, $\rho_{1}$. The values obtained for $N_{0}$ in previous experiments ${ }^{2}$ are $0.73,0.95$, and $0.98 \times 10^{15} \mathrm{~cm}^{-2}$. We choose an average of $N_{0}=0.9 \times 10^{15} \mathrm{~cm}^{-2}$. The third term on the righthand side of Eq. (4) represents the energy associated with the undercooling of a liquid film to a temperature below the melting point. The undercooling energy is responsible for the temperature dependence of $\gamma_{\text {slv }}(N, \Theta, T)$. First, for fixed $N$, the undercooling energy increases linearly with decreasing temperature, and, second, it makes the thickness of the liquidlike film a function of temperature. The fourth term in Eq. (4) contains the excess interfacial free energy $\Delta \gamma(\Theta)$ [see Eq. (1)], multiplied by a correction factor which accounts for the fact that the liquidlike film has a finite thickness at temperatures below $T_{m}$. In Eq. (4) we have neglected the entropic contributions to the temperature dependence of $\gamma_{\mathrm{sv}}(\Theta)$, $\gamma_{\mathrm{sl}}(\Theta)$, and $\gamma_{\mathrm{lv}}$ (see also Sec. VIII).

The equilibrium melted-film thickness as a function of temperature and surface orientation, $N^{\mathrm{eq}}(T, \Theta)$, is obtained by minimizing Eq. (4) with respect to the number of positionally disordered atoms per unit area, $N$. This yields the following expression for the growth of the melted surface film: ${ }^{1,2}$

$$
N^{\mathrm{eq}}(\Theta, T)=N_{0} \ln \left[\frac{T_{m} \Delta \gamma(\Theta)}{N_{0} L_{m}\left(T_{m}-T\right)}\right] .
$$

Substituting this equilibrium film thickness into Eq. (4), and using the assumption $\gamma_{\mathrm{sl}}(\Theta)=0.1 \gamma_{\mathrm{sv}}(\Theta)$, we arrive at the effective free energy for melted surfaces as a function of temperature:

$$
\gamma_{\mathrm{slv}}(\Theta, T)=0.1 \gamma_{\mathrm{sv}}(\Theta)+\gamma_{\mathrm{lv}}+N_{0} L_{m}\left(1-T / T_{m}\right)\left\{1+\ln \left[\frac{T_{m} \Delta \gamma(\Theta)}{N_{0} L_{m}\left(T_{m}-T\right)}\right]\right\}
$$

For each $T \leq T_{m}$, the facet orientations $\Theta_{d^{+}}, \Theta_{d^{-}}, \Theta_{m}+$, and $\Theta_{m}{ }^{-}$, are obtained from the double-tangent construction in the $\beta$ plot of $\gamma_{\mathrm{sv}}(\Theta)$ and $\gamma_{\mathrm{slv}}(\Theta, T)$. Mathematically, the double-tangent construction is expressed by the following differential equations:

$$
\begin{aligned}
& \frac{\gamma_{\mathrm{slv}}\left(\Theta_{m}, T\right)}{\cos \left(\Theta_{m}\right)}-\frac{\gamma_{\mathrm{sv}}\left(\Theta_{d}\right)}{\cos \left(\Theta_{d}\right)} \\
& \quad=\left.\left\{\tan \left(\Theta_{m}\right)-\tan \left(\Theta_{d}\right)\right\} \frac{\partial\left(\gamma_{\mathrm{sv}}(\Theta) / \cos (\Theta)\right)}{\partial \tan (\Theta)}\right|_{\Theta_{d}},
\end{aligned}
$$

$\left.\frac{\partial\left(\gamma_{\mathrm{sv}}(\Theta) / \cos (\Theta)\right)}{\partial \tan (\Theta)}\right|_{\Theta_{d}}=\left.\frac{\partial\left(\gamma_{\mathrm{slv}}(\Theta, T) / \cos (\Theta)\right)}{\partial \tan (\Theta)}\right|_{\Theta_{m}}$.

One could parametrize the orientation dependence of $\gamma_{\text {sv }}(\Theta)$ measured for $\mathrm{Pb}$ by Heyraud and Métois, and try to solve the problem analytically. However, this approach fails because the logarithmic term in $\gamma_{\text {slv }}(\Theta, T)$ makes the set of differential equations (7) strongly nonlinear. Therefore, we have determined the facet orientations numerically.

The cause of the temperature dependence of the facet orientations is illustrated by the difference between the $\beta$ plot for $T=T_{m}$ (Fig. 2) and that for $T=590 \mathrm{~K}$ (Fig. 10), which is obtained by using Eq. (6). The free-energy curve for dry surfaces, $\gamma_{\mathrm{sv}}(\Theta) / \cos (\Theta) \gamma_{\mathrm{sv}}^{(111)}$, is independent of temperature and is therefore the same in both figures. As the temperature is lowered from 600.7 (Fig. 2) to $590 \mathrm{~K}$ (Fig. 10), the effective free-energy curve for melted surfaces, $\gamma_{\text {slv }}(\Theta, T) / \cos (\Theta) \gamma_{\text {sv }}^{(111)}$, shifts upwards due to the undercooling energy and becomes steeper. As a consequence, the orientations of the dry and melted facets for both positive and negative miscut angles shift away from the (111) orientation, while the dry and the melted facet orientations approach each other. The results of the calculation are shown in Fig. 11. The orientations of the dry and melted facets are indicated by the dashed and solid curves, respectively. For miscut angles toward the (110) surface (positive angles), the two facet orientations $\Theta_{d}+$ and $\Theta_{m}+$ merge at the (110) orientation at $506 \pm 5 \mathrm{~K}$. At that temperature $\mathrm{Pb}(110)$ starts to melt, i.e., the calculated free energy for melted $\mathrm{Pb}(110)$ is equal to the calculated energy for dry $\mathrm{Pb}(110)$, whereas for all other surface orientations the melted energy is higher than the dry energy. Below $506 \mathrm{~K}$, the melted surface free energy is higher than that for a dry surface for all surface orientations. $\mathrm{Pb}(110)$ is the surface orientation which starts to melt at the lowest temperature. Above this temperature, surface-melting-induced faceting 


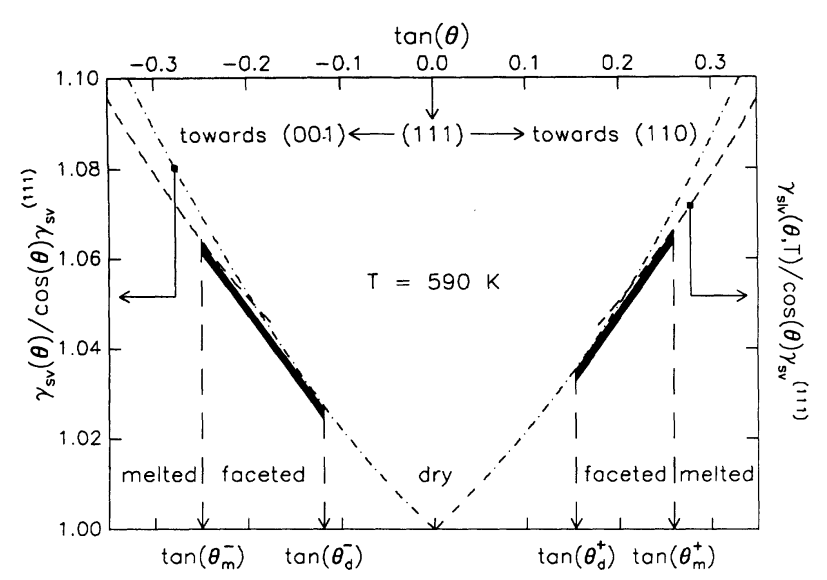

FIG. 10. $\beta$ plot of the calculated effective surface free energies at $590 \mathrm{~K}$. The normalized effective free energy for melted surfaces, $\gamma_{\mathrm{slv}}(\Theta, T) / \cos (\Theta) \gamma_{\mathrm{sv}}^{(111)}$ (dashed curve), shifts upwards with respect to the free energy for dry surfaces, $\gamma_{\mathrm{sv}}(\Theta) / \cos (\Theta) \gamma_{\mathrm{sv}}^{(111)}$ (dash-dotted curve), with decreasing temperature. As a result, the facet orientations which are obtained from the double-tangent construction (solid-line segments) move away from the (111) orientation, and the ranges of orientations where surface-melting-induced faceting occurs shrink with decreasing temperature. Note that there is a range of orientations for which $\gamma_{\text {slv }}(\Theta, T)$ is not defined, because the free energy for melted surfaces [Eq. (6)] is only meaningful when the solution of Eq. (5) is positive.

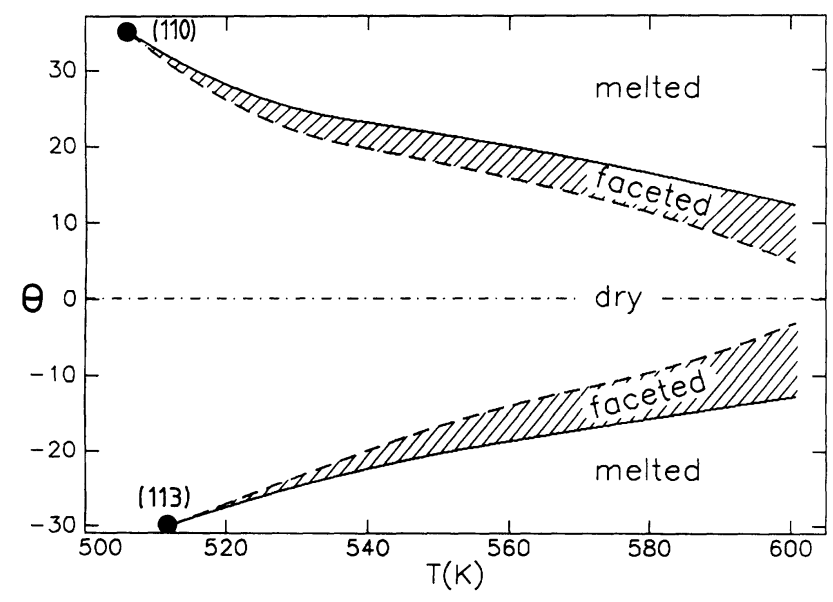

FIG. 11. Temperature dependence of the facet orientation angles obtained from the model calculations discussed in Sec. VI. The solid and dashed curves show the melted and dry facet orientations, respectively. Surface-melting-induced faceting occurs for orientations in the shaded areas, between dry and melted orientations. Between 500 and $520 \mathrm{~K}$, the melted and dry facet orientations merge, both for positive and negative orientation angles, at the (110) orientation and near to the (113) orientation, respectively, as indicated by the large dots. Below this temperature, there is no surface melting and, hence, no surface-melting-induced faceting. occurs. Toward the $(001)$ orientation, the facet orientation angles merge at approximately the (113) orientation at $512 \pm 5 \mathrm{~K}$. The two points in Fig. 11 where the facet angles disappear, indicated by large dots, can be considered as critical points. Above these critical temperatures, regions of orientational phase coexistence appear.

The high-temperature part of the calculation results has also been plotted in Fig. 9, in order to confront the model with the experimentally obtained orientation angles. The shaded area around the model curves indicates the uncertainty in our calculated results. The doubletangent construction is very sensitive to small errors in the input data. In Ref. 31 the accuracy in the relative surface free energies $\gamma(\Theta) / \gamma^{(111)}$ was claimed to be \pm 0.0016 . We estimate that this leads to an accuracy in the values we obtain from the double-tangent construction of $\pm 2^{\circ}$. The order of magnitude for the facet orientations predicted by the model agrees rather well with the experimental results. Like the experiment, the model indicates that the facet orientations change no more than a few degrees over the experimentally addressed temperature range. Although the difference between $\Theta_{d^{-}}$and $\Theta_{d^{+}}$is predicted correctly by the calculation, the asymmetry, i.e., the difference between the absolute values of $\Theta_{d^{-}}$and $\Theta_{d^{+}}$, comes out the wrong way around.

\section{IMPLICATIONS FOR THE EQUILIBRIUM SHAPE}

In this section we discuss the observed equilibrium shape of $\mathbf{P b}$ crystallites at high temperature ${ }^{17,18}$ in terms of surface-melting-induced faceting. Heyraud and Métois ${ }^{17}$ have reported a slope discontinuity, i.e., a sharp edge, of approximately $16^{\circ}$ near the plane (111) facet on the shape of small lead particles close to the bulk melting temperature. A slope discontinuity implies the absence of a range of surface orientations from the equilibrium shape. The absent range should be precisely the range of orientations that is identified as unstable by the doubletangent construction. In Refs. 16 and 17 it was proposed that the slope discontinuity is caused by the coexistence of melted and dry orientations. The limiting orientations on both sides of the edge should then correspond to the facet orientations $\Theta_{d}$ and $\Theta_{m}$, which we have measured for macroscopic surfaces. The angle of $16^{\circ}$ which has been measured in Ref. 17 is probably not the slope discontinuity itself, i.e., the difference between $\Theta_{d}$ and $\Theta_{m}$, but rather the limiting orientation at the melted side of the slope discontinuity, $\Theta_{m}$, indicated in Fig. 12. Only when $\Theta_{d}$ was zero would $\Theta_{m}$ be equal to the slope discontinuity. In Ref. 17, the azimuth for which $\Theta_{m}$ has been measured is not specified. However, we can still compare the result of Heyraud and Métois to our result, since our measurements indicate that $\Theta_{m}$ does not depend strongly on the miscut direction. For temperatures around $600 \mathrm{~K}$ we obtain absolute values of $\Theta_{m}$ close to $14.5^{\circ}$ in both miscut directions, which is in agreement with the equilibrium-shape observation of $\sim 16^{\circ} .{ }^{17}$ Heyraud and Métois do not discuss whether the orientation angle at the other side of the slope discontinuity, which should correspond to our $\Theta_{d}$, is $0^{\circ}$ or has a 


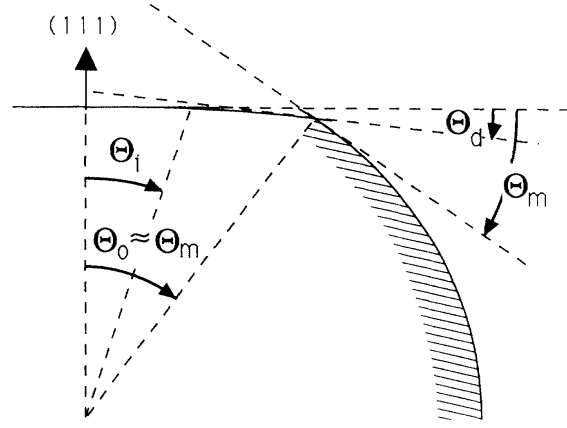

FIG. 12. Sketch of the local equilibrium shape of $\mathrm{Pb}$ near the bulk melting temperature, expected from our results. The planar dry (111) facet is smoothly connected to a region of stable dry vicinal orientations. This region is connected by a sharp edge to the region of surface-melted orientations (shaded). This slope discontinuity arises from the instability of the intermediate vicinal orientations. The limiting orientations on both sides of the edge are $\Theta_{d}$ and $\Theta_{m}$. The angles $\Theta_{i}$ and $\Theta_{o}$ are explained in Sec. VII.

nonzero value. Probably such subtle orientation differences are difficult to measure from the electronmicroscopy images. According to the model described in Sec. VI, the lowest temperature for surface-meltinginduced faceting is approximately $510 \mathrm{~K}$. This implies that the equilibrium shape of $\mathrm{Pb}$ should exhibit a sharp edge for all temperatures between 510 and $600.7 \mathrm{~K}$. However, in Ref. 17 the sharp edge was reported only for temperatures above $580 \mathrm{~K}$.

Equilibrium-shape measurements for $\mathrm{Pb}$ at high temperatures have also been performed by Pavlovska, Faulian, and Bauer. ${ }^{18}$ The electron-microscopy images in Ref. 18 have revealed a bright ring around the (111) plane above $580 \mathrm{~K}$. The interpretation given in Ref. 18 was that there is a ring of rough surface orientations, in between the flat (111) facet and the surrounding surfacemelted orientations. The observations of Ref. 18 can be explained qualitatively by surface-melting-induced faceting. At high temperatures, there is always a range of vicinal surfaces, with orientations between (111) and $\Theta_{d}$, which remains dry. Because of the large step-step distances, these vicinal surfaces are already rough far below $T_{m}$. They could form the observed bright ring around the flat (111) plane. The inner perimeter of this ring would then be the border between rough but dry vicinals and the dry (111) orientation itself, while the outer perimeter would be the boundary between dry vicinals and melted orientations. According to this interpretation, the outer perimeter of the ring should be the location of the slope discontinuity observed by Heyraud and Métois. ${ }^{17}$ Pavlovska, Faulian, and Bauer do not mention a slope discontinuity. ${ }^{18}$ In Ref. 18 the outer and inner radii of the ring have been monitored as a function of temperature between 560 and $600 \mathrm{~K}$. These radii have been quantified in terms of half-angles $\Theta_{o}$ and $\Theta_{i}$. We propose that these half-angles relate to the equilibrium shape as indicated in Fig. 12. Because the free energy of the surface-melted regions is nearly isotropic, the melted part of the equilibrium shape is almost spherical and the outside half-angle $\Theta_{o}$ should be almost equal to the orientation of $\Theta_{m}$. The data of Ref. 18 extrapolate to a value of $\Theta_{o}=13.5^{\circ}$ at $T_{m}$, which agrees with our result of $\Theta_{m}{ }^{+}=14.7^{\circ} \pm 1.4^{\circ}$ and $\Theta_{m^{-}}=-14.5^{\circ} \pm 1.2^{\circ}$ close to the bulk melting point. Pavlovska, Faulian, and Bauer found an increase of $\Theta_{o}$ with decreasing temperature, which is qualitatively in agreement with both our observations and our calculations. The inside half-angle $\Theta_{i}$ is not affected by the surface-melting-induced faceting, but reflects the ratio of the specific free energy of the steps created by the miscut and the specific free energy $\gamma^{(111)}$ of the (111) facet. ${ }^{7} \mathrm{~A}$ high step free energy leads to large values for $\Theta_{i}$.

\section{DISCUSSION}

Löwen has applied the Wulff construction to the situation in which some surface orientations melt. ${ }^{33} \mathrm{He}$ has calculated the temperature-dependent equilibrium shape of $\mathrm{Pb}$ particles close to the triple point. The angles he has obtained for the surface orientations on both sides of the sharp edge on the equilibrium shape are a few degrees different from the results of our model and shows a weaker temperature dependence. In principle, Löwen's calculation should yield the same orientations as the procedure described in Sec. VI because it makes use of the same expression for the temperature dependence of the effective surface free energy $\gamma_{\text {slv }}$ [Eq. 6)], and because the doubletangent construction that we use to obtain $\Theta_{d}$ and $\Theta_{m}$ is implied by the Wulff construction used by Löwen. ${ }^{7,8}$ However, there are three differences between Löwen's calculation and ours. First, he has explicitly taken into account the (entropic) temperature dependence of the interfacial free energies, $\gamma_{\mathrm{sv}}$ (Ref. 32) and $\gamma_{\mathrm{lv}}{ }^{34}$ The variation of these energies with temperature is approximately $-1 \times 10^{-4} \mathrm{~J} \mathrm{~m}^{-2} \mathrm{~K}^{-1}$. However, the resulting change in the difference between the free energy for dry surfaces $\gamma_{\text {sv }}(\Theta, T)$ and the effective free energy for melted surfaces $\gamma_{\text {slv }}(\Theta, T)$ is only of the order of $1 \times 10^{-5} \mathrm{~J} \mathrm{~m}^{-2} \mathrm{~K}^{-1}$. Hence the temperature dependence of $\Theta_{d}$ and $\Theta_{m}$ is not altered significantly by the temperature dependence of the interfacial free energies. Second, in Ref. $33 N_{0}$ has been set to $0.732 \times 10^{15} \mathrm{~cm}^{-2}$. Naturally, this has resulted in a somewhat weaker temperature dependence than that for our choice of $N_{0}=0.9 \times 10^{15} \mathrm{~cm}^{-2}$. The small differences between the results of Löwen and our calculation arise mainly from the third difference between his calculation and ours. In Ref. 33, the orientation dependence of $\gamma_{\mathrm{sv}}$ was obtained from interpolation and extrapolation of equilibrium-shape data measured by Heyraud and Métois at temperatures up to $573 \mathrm{~K} .{ }^{31}$ The assumption that the equilibrium shape at high temperature still reflects the orientation dependence of the solid vapor interface is questionable. Above $510 \mathrm{~K}$ several surface orientations are already melted. The orientation dependence of the surface free energy measured above $510 \mathrm{~K}$ in Ref. 31 is therefore a mixture of $\gamma_{\text {sv }}$ (for the nonmelted orientations) and $\gamma_{\text {slv }}$ (for the melted-surface orientations). We have preferred to use the surface free energies 
measured by Heyraud and Métois at $473 \mathrm{~K}$, where there is no surface melting.

From the MEIS measurements, we find $\left|\Theta_{d^{+}}\right|<\left|\Theta_{d^{-}}\right|$. This is opposite to the predictions from our model, which uses the orientation dependence of the surface free energies at $473 \mathrm{~K}$ from Ref. 31. In Figs. 2 and 10 , one can see that $\Theta_{d}$ is small when $\gamma_{\text {sv }}$ is a steep function of surface orientation. In the data for $473 \mathrm{~K}$ of Ref. 31, the slope of the free-energy curve for miscuts toward the (001) orientation is steeper than the slope toward the (110) orientation. However, for $573 \mathrm{~K}$ the data in Ref. 31 show the opposite behavior: at $573 \mathrm{~K}$ the slope of the free-energy plot is steeper toward the (110) direction than toward the (001) direction. The latter is consistent with our observation that the dry facet orientation for positive miscut angles lies closer to the (111) orientation than that for negative miscut angles. As the slope of the surface freeenergy curve is proportional to the step free energy, 7,16 our observations, and those of Heyraud and Métois, indicate that the entropy of steps toward (110) is lower than that of steps toward (001). In the literature on the equilibrium shape of small $\mathrm{Pb}$ particles, ${ }^{17,18,31}$ an azimuthal asymmetry of the shape of the (111) facet has not been mentioned. In view of the symmetry of the (111) plane, the difference we find between $\left|\Theta_{d^{+}}\right|$and $\left|\Theta_{d^{-}}\right|$should be accompanied by a triangular symmetry of the inner perimeter of the rings observed by Pavlovska, Faulian, and Bauer. ${ }^{18}$ The outer perimeter should be close to a true circle, since $\mid \Theta_{m}+$ and $\left|\Theta_{m}-\right|$ are almost equal.

Bilalbegović, Ercolessi, and Tosatti have performed molecular-dynamics simulations of vicinals of $\mathrm{Pb}(111)$, with an orientation in the $[11 \overline{2}]$ zone. ${ }^{13}$ The simulation cells were approximately $100 \AA$ long in the direction perpendicular to the steps, and four atoms wide parallel to the steps. A glue potential was used to describe the atomic interactions. The azimuthal direction of the miscut of the simulated vicinals was $90^{\circ}$ rotated with respect to that of the vicinals in the [1 10 ] zone discussed by us. Although these azimuthal directions are inequivalent, we may compare the qualitative features of the simulation results with our experimental findings. At $T=0.97 T_{m}$, the simulated unit cells facet with $\Theta_{m}$ values of $18^{\circ}$ and $27^{\circ}$, for $\mathrm{Pb}(534)$ and $\mathrm{Pb}(423)$, respectively. Apparently, the lever rule does not work properly for these small facets. In Ref. 13 this was attributed to the limited size of the simulation cells. Bilalbegović, Ercolessi, and Tosatti assumed that the stable dry facets have the (111) orientation, ${ }^{13}$ i.e., $\Theta_{d}=0^{\circ}$, although they reported the occurrence of isolated steps on the dry facets in some simulations. This qualitative difference with our result of nonzero $\Theta_{d}$ values is probably also due to the small size of the simulation cells. The average step-step distance on a $3^{\circ}$ vicinal would be $55 \AA$, so that a substantially larger unit cell would be required to discriminate this vicinal orientation from the (111) plane itself. Another source for the differences between the simulations and our experimental findings could be the energy associated with the line of contact between two neighboring facets, which necessarily becomes important when microscopic facets are considered.
We observe that macroscopic vicinal surfaces decompose into coexisting dry and melted facets at high temperature. The MEIS spectra are insensitive to the size of the facets. However, we can give upper and lower estimates. The fact that the analysis based on linear superpositions works properly, implies (i) that there are enough facets within our beam spot to measure both types of facets with the correct average weights, and (ii) that the finite size of the facets has no effect on the melted film thicknesses. The error bars in Fig. 6 indicate that, for sufficiently long counting times, we can obtain an accuracy in the fitted fractions which is much better than $10 \%$. This means that our beam spot integrates over at least $10^{2}$ facet pairs. With a beam diameter of approximately $1 \mathrm{~mm}$, we arrive at an upper limit for the facet sizes of $10 \mu \mathrm{m} .{ }^{35}$ As the melted-film thicknesses at the highest experimental temperature are on the order of 1 $\mathrm{nm}$, we estimate that finite-size effects come into play at sizes of about $10 \mathrm{~nm}$. Hence we estimate that the facets have a size between $10 \mathrm{~nm}$ and $10 \mu \mathrm{m}$. This is precisely the length scale which is investigated in the equilibrium shape measurements of Refs. 17 and 18.

\section{CONCLUSIONS}

We have experimentally demonstrated that there are ranges of vicinal orientations around $\mathrm{Pb}(111)$ which exhibit surface-melting-induced faceting: these vicinal surfaces decompose into coexisting dry and melted facets. The faceting is not restricted to temperatures close to the bulk melting point, but occurs over a temperature range, which extends at least from $589.6 \mathrm{~K}$ to $T_{m}$. Within the [110] zone, the faceting occurs both for vicinal surfaces with a miscut angle toward (110) and for surfaces with a miscut angle toward (001). Close to the melting point, the orientation of the melted facets is approximately $14.5^{\circ}$ away from the (111) orientation for both miscut directions. At this temperature, the orientation of the dry facets makes an angle of $3.1^{\circ} \pm 0.6^{\circ}$ with respect to (111) for vicinals oriented toward (110), and $7.3^{\circ} \pm 0.8^{\circ}$ for vicinal orientations toward (001). Above $593.8 \mathrm{~K}$, the dry facet orientations hardly change with temperature.

We have theoretically described the phenomenon of surface-melting-induced faceting and its temperature dependence in terms of the orientation and temperature dependence of the free energies for dry and melted $\mathrm{Pb}$ surfaces. The temperature dependence of the facet orientations has been computed within a model which is based on a Landau expression for the effective free energy of melted surfaces. The model correctly describes the order of magnitude of the facet orientations and that of their variation with temperature. It does not correctly predict the observed azimuthal anisotropy in the dry facet orientation, probably as a consequence of the neglect of the temperature dependence of $\gamma_{\mathrm{sv}}(\Theta) / \gamma_{\mathrm{sv}}^{(111)}$.

Finally, we have shown that the surface-meltinginduced faceting is reflected in the equilibrium shape of $\mathrm{Pb}$ microcrystallites. ${ }^{17,18}$

Faceting induced by surface melting should be regarded as a general phenomenon directly resulting from the coexistence of melting and nonmelting surface orienta- 
tions. The orientational phase separation observed on $\mathbf{P b}$ is expected to occur for a variety of materials, such as most metals and many molecular crystals.

\section{ACKNOWLEDGMENTS}

The authors wish to thank $H$. Löwen for valuable correspondence on the calculations of Ref. 33. J. F. van der Veen is gratefully acknowledged for carefully reading the manuscript. This work is part of the research program of the Stichting voor Fundamenteel Onderzoek der Materie (Foundation for Fundamental Research on Matter) and was made possible by financial support from the Nederlandse organisatie voor Wetenschappelijk Onderzoek (Netherlands Organization for Scientific Research).
${ }^{1}$ J. W. M. Frenken and H. M. van Pinxteren, in The Chemical Physics of Solid Surfaces and Heterogeneous Catalysis, Vol. 7: Phase Transitions and Adsorbate Restructuring at Metal Surfaces, edited by D. A. King and D. P. Woodruff (Elsevier, Amsterdam, 1993), Chap. 8.

${ }^{2}$ B. Pluis, A. W. Denier van der Gon, J. F. van der Veen, and A. J. Riemersma, Surf. Sci. 239, 265 (1990).

${ }^{3}$ J. W. M. Frenken, P. M. J. Marée, and J. F. van der Veen, Phys. Rev. B 34, 7506 (1986).

${ }^{4}$ A. W. Denier van der Gon, R. J. Smith, J. M. Gay, D. J. O'Connor, and J. F. van der Veen, Surf. Sci. 227, 143 (1990).

${ }^{5}$ B. Pluis, D. Frenkel, and J. F. van der Veen, Surf. Sci. 239, 282 (1990).

${ }^{6}$ C. Herring, Phys. Rev. 82, 87 (1951).

${ }^{7}$ M. Wortis, in Chemistry and Physics of Solid Surfaces, edited by R. Vanselow and R. F. Howe (Springer, Berlin, 1988), Vol. 7, p. 367.

${ }^{8}$ N. Cabrera, Surf. Sci. 2, 320 (1964).

${ }^{9}$ M. Drechsler, Surf. Sci. 266, 1 (1992).

${ }^{10}$ M. Flytzani-Stepanopoulos and L. D. Schmidt, Prog. Surf. Sci. 9, 83 (1979).

${ }^{11}$ E. D. Williams and N. C. Bartelt, Science 251, 393 (1991).

${ }^{12}$ E. D. Williams and N. C. Bartelt, Ultramicroscopy 31, 36 (1989).

${ }^{13}$ G. Bilalbegović, F. Ercolessi, and E. Tosatti, Europhys. Lett. 17, 333 (1992).

${ }^{14} \mathrm{G}$. Bilalbegović, F. Ercolessi, and E. Tosatti, Surf. Sci. 280, 335 (1993).

${ }^{15}$ H. M. van Pinxteren and J. W. M. Frenken, Europhys. Lett. 21, 43 (1993).

${ }^{16}$ P. Nozières, J. Phys. 50, 2541 (1989).

${ }^{17}$ J. C. Heyraud, J. J. Métois, and J. M. Bermond, J. Cryst. Growth 98, 355 (1989).
${ }^{18}$ A. Pavlovska, K. Faulian, and E. Bauer, Surf. Sci. 221, 233 (1989).

${ }^{19}$ J. W. Herman and H. E. Elsayed-Ali, Phys. Rev. Lett. 69, 1228 (1992).

${ }^{20}$ H. M. van Pinxteren and J. W. M. Frenken, Surf. Sci. 275, 383 (1992).

${ }^{21}$ A. W. Denier van der Gon, J. M. Gay, J. W. M. Frenken, and J. F. van der Veen, Surf. Sci. 241, 335 (1991).

${ }^{22}$ A. A. Chernov and L. V. Mikheev, Phys. Rev. Lett. 60, 2488 (1988).

${ }^{23} \mathrm{E}$. Tosatti (private communication).

${ }^{24}$ G. Bilalbegović, F. Ercolessi, and E. Tosatti, Europhys. Lett. 18, 163 (1992).

${ }^{25}$ A. Bartolini, F. Ercolessi, and E. Tosatti, Phys. Rev. Lett. 63, 872 (1989).

${ }^{26}$ H. P. Bonzel, U. Breuer, B. Voigtländer, and E. Zeldov, Surf. Sci. 272, 10 (1992).

${ }^{27}$ E. E. Gruber and W. W. Mullins, J. Phys. Chem. Solids 28, 875 (1967)

${ }^{28}$ This definition is opposite to that used in Ref. 2.

${ }^{29}$ J. F. van der Veen, Surf. Sci. Rep. 5, 199 (1985).

${ }^{30}$ J. F. Ziegler, J. P. Biersack and U. Littmark, in The Stopping and Ranges of Ions in Solids, edited by J. F. Ziegler (Pergamon, New York, 1985), Vol. 1.

${ }^{31}$ J. C. Heyraud and J. J. Métois, Surf. Sci. 128, 334 (1983).

${ }^{32}$ A. R. Miedema, Z. Metallkd. 69, 287 (1978).

${ }^{33}$ H. Löwen, Surf. Sci. 234, 315 (1990).

${ }^{34}$ A. R. Miedema and R. Boom, Z. Metallkd. 69, 183 (1978).

${ }^{35}$ In these estimates we assume the faceting to be largely one dimensional. The length of the facets along the close-packed [110] step direction is probably much larger than that perpendicular to the steps. 


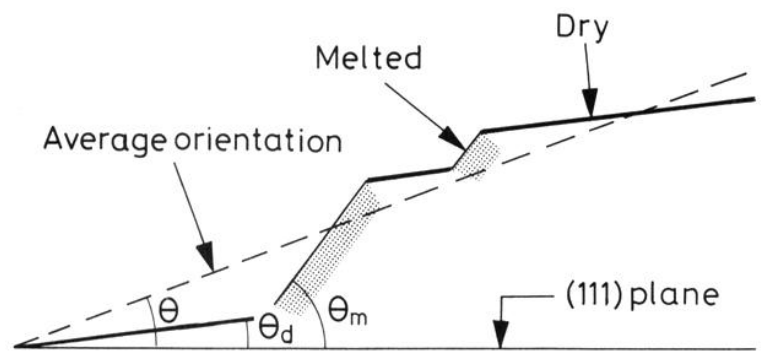

FIG. 1. Schematic picture of surface-melting-induced faceting. The surface, with initial miscut angle $\Theta$ has decomposed into dry and melted facets with orientations $\Theta_{d}$ and $\Theta_{m}$, respectively. 


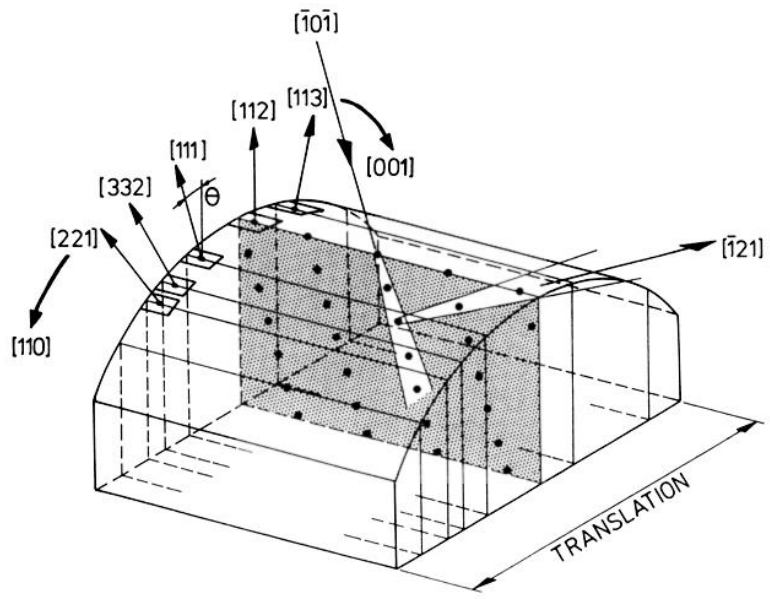

FIG. 3. The shadowing and blocking geometry in the (111) scattering plane of a cylindrical single crystal of $\mathrm{Pb}$. The crystal exposes a range of surface orientations around the (111) orientation. Surfaces with a positive orientation angle $\Theta$ are miscut, with respect to (111), toward the (110) orientation, whereas surfaces with a negative $\Theta$ are oriented toward (001). 


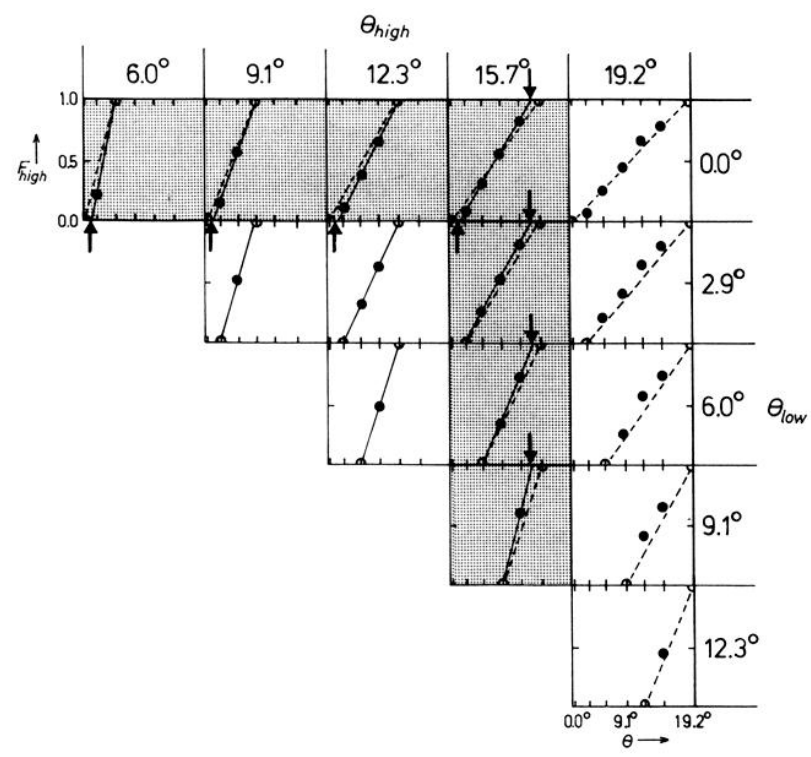

FIG. 6. Results of fitting the energy spectra measured at $600.65 \mathrm{~K}$ for positive miscut angles by a linear combination of two other spectra. For each combination of $\Theta_{\text {low }}$ and $\Theta_{\text {high }}$ (one panel in the table) the best fits to the spectra for orientations $\Theta$ between those angles are obtained for the fractions $F_{\text {high }}$ (solid circles) of the high-angle spectrum. The open circles indicate the trivial result of $F_{\text {high }}=0$ for $\Theta=\Theta_{\text {low }}$, and $F_{\text {high }}=1$ for $\Theta=\Theta_{\text {high }}$. The fractions for each combination of $\Theta_{\text {low }}$ and $\Theta_{\text {high }}$ have been plotted as a function of $\tan \left(\Theta+19.5^{\circ}\right.$ ) (see text and Fig. 7), on a scale ranging from $\tan \left(0^{\circ}+19.5^{\circ}\right)$ to $\tan \left(19.2^{\circ}+19.5^{\circ}\right)$. Spectra have been measured for $0.0^{\circ}, 2.9^{\circ}$, $6.0^{\circ}, 9.1^{\circ}, 12.3^{\circ}, 15.7^{\circ}$, and $19.2^{\circ}$, corresponding to the tick marks on the horizontal axes. The geometrical condition [Eq. (3)] is indicated by the dashed straight lines. The solid straight lines are fits to the fractions obtained for orientations within the range of surface-melting-induced faceting. The arrows pointing up locate the orientation of the stable dry facets $\Theta_{d}$, and the arrows pointing down locate the orientation of the melted facets $\Theta_{m}$. The shading of the panels with $\Theta_{\text {low }}=0.0^{\circ}$ and $\Theta_{\text {high }}=15.7^{\circ}$ is discussed in the text. 


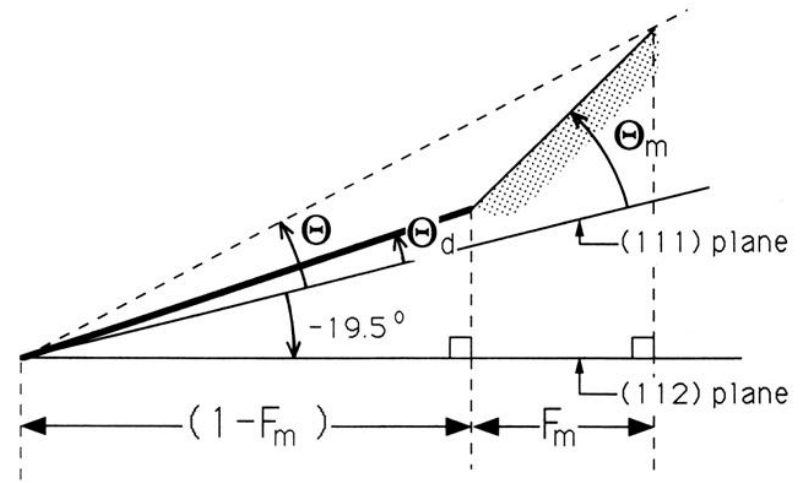

FIG. 7. Illustration of the lever rule [Eq. (3)], which expresses that the average orientation of a faceted surface is fixed. Indicated are the fraction of melted surface area projected on the (112) plane, $F_{m}$, and the fraction of dry surface area projected on the (112) plane, $1-F_{m}$. In the fitting of the MEIS spectra this is the relevant projection, since the scattering plane was perpendicular to the (112) plane. 


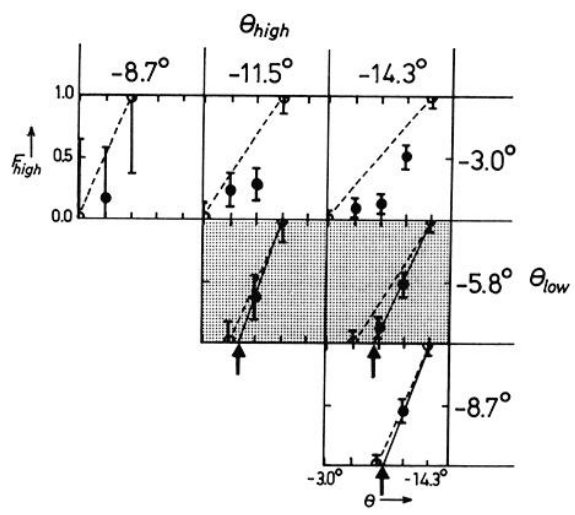

FIG. 8. Results from fitting the energy spectra measured at 593.8 $\mathrm{K}$ for negative miscut angles. The layout of this figure corresponds to that of Fig. 6. Spectra have been measured for $-3.0^{\circ},-5.8^{\circ},-8.7^{\circ},-11.5^{\circ}$, and $-14.3^{\circ}$. The geometrical condition is again indicated by the dashed straight line. The error bars depict the statistical accuracy in the fractions $F_{\text {high }}$ obtained from fitting the energy spectra with each other. 


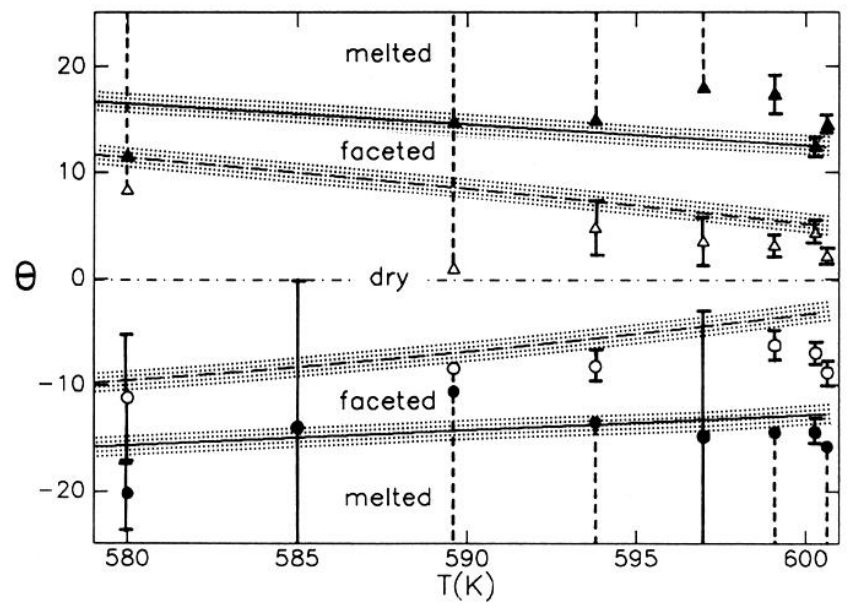

FIG. 9. Experimentally determined facet orientation angles as a function of temperature. The triangles are the results for vicinals with a miscut toward the (110) orientation, the circles for vicinals with a miscut toward the $(001)$ orientation. Open symbols depict the dry facet orientations, solid symbols the melted facet orientations. Dashed error bars indicate that the depicted angle is either a lower or an upper limit for the facet orientation. The curves are part of the model-calculation results of Sec. VI (see Fig. 11). The dashed curves correspond to the predictions for the dry facet angles, the solid curves to those for the melted facet angles. The shading around these curves indicates the estimated accuracy of the model. 